
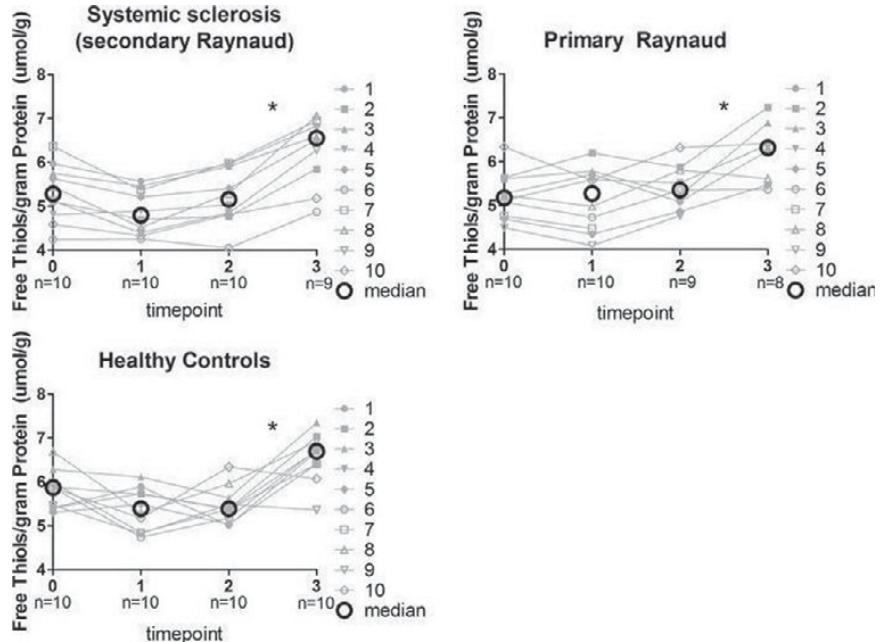

Figure 1. Free thiols per gram protein in all groups

${ }^{\star} p<0.005$ between timepoint 2 and 3

Although these findings need further study, they may suggest activation of ubiquitous antioxidant defense mechanisms during cooling and/or rewarming and should be explored for future use as a potential therapeutic target in RP. References:

[1] van Roon AM, et. al. Rheumatology (Oxford). 2016 Jun;55(6):1083-90.

[1] 2 Koning AM, et al. Pharmacol Res 2016 Sep;111:452-458.

[2] 3 Umbrello M, et al. J Physiol 2014 Mar 1;592(5):1061-1075.

Disclosure of Interest: None declared

DOI: 10.1136/annrheumdis-2017-eular.5972

\section{FRI0367 NEW AUTOIMMUNE TARGETS IN IDIOPATHIC INFLAMMATORY MYOPATHIES - AN ANTIGEN BEAD ARRAY APPROACH}

A. Notarnicola ${ }^{1}$, C. Hellstrom ${ }^{2}$, C. Mattsson ${ }^{2}$, E. Andersson ${ }^{2}$, H. Idborg ${ }^{1}$, E. Jemseby ${ }^{1}$, M. Neiman ${ }^{2}$, P.-J. Jakobsson ${ }^{1}$, P. Nilsson ${ }^{2}$, I.E. Lundberg ${ }^{1}$. ${ }^{1}$ Department of Medicine, Rheumatology Unit, Karolinska University Hospital and Karolinska Institutet; ${ }^{2}$ SciLifeLab, KTH - Royal Institute of Technology, Solna, Stockholm, Sweden

Background: The Idiopathic Inflammatory Myopathies (IIM) is a group of rare systemic inflammatory diseases characterized by severe organ involvement and premature mortality. Several myositis specific auto-antibodies (MSAs) have been recognized and associated with specific clinical manifestations and prognosis, still many patients are autoantibody negative. Identification of new autoimmune targets will be helpful in improving diagnosis, better stratifying into subgroups, prediction of prognosis, tailoring treatment and to understand underlying biological pathways.

Objectives: To identify new autoimmune targets in IIM by antigen bead array (1). Methods: A bead array with 354 antigens was used to explore the autoimmune reactivity in 881 plasma samples from patients with IIM $(\mathrm{N}=225)$, Systemic Lupus Erythematosus (SLE) $(\mathrm{N}=350)$ and population controls $(\mathrm{N}=306)$. The antigens were selected from initial screenings of 160 SLE-samples on a total of 5760 antigens on planar arrays, and a first verification bead array with 355 antigens. The IIM samples represented three groups of patients with distinct diagnoses: Dermatomyositis (DM, N=83), Polymyositis ( $\mathrm{PM}, \mathrm{N}=111$ ) and Inclusion Body Myositis (IBM, N=31), who were regularly followed at the Rheumatology Unit of the Karolinska University Hospital from January 2003 until March 2014. Based on 2 possible levels of cutoff, each sample was classified as reactive to each single antigen $(\mathrm{Ag})$ at low or high cut off or non-reactive.

Results: In general, depending on the cutoff stringency, $86-88 \%$ of the 354 selected antigens showed reactivity in at least one sample with no difference between IIM, SLE and controls. Comparing PM, DM, IBM according to the number of samples which showed reactivity towards each single Ag, reactivity at high cut off towards NADH dehydrogenase $1 \alpha$ subcomplex 11 (NDUFA11), poly(A) RNA polymerase D4 (PAPD4), CD163, I(3)mbt-like 1 (L3MBTL1) and calcium release-activated calcium modulator 2 (ORAI2) was discovered with higher frequencies in the IBM samples compared to PM and DM. In the group of IIM patients testing negative for all the known MSAs increased reactivity at high cut off was observed towards E3 ubiquitin protein ligase 2 (SIAH2), leiomodin 2 (LMO2) and RAD23 homolog A (RAD23A). In the group of IIM patients with history of malignancy and no evidence for anti-p155/140 antibodies the antigens early B-cell factor 2 (EBF2), POU class 6 homebox 1 (POUF61) and growth differentiation factor 7 (GDF7) revealed high serum reactivity. In IIM patients with interstitial lung disease increased reactivity at high cut off was found towards zinc finger protein 688 (ZNF688) and prostaglandin D2 receptor (PTGDR). A high frequency of known target reactivities (MSAs) was also confirmed.

Conclusions: Reactivity towards autoantigens corresponding to human proteins was present in plasma samples from IIM, controls, and SLE. Potentially new autoimmune targets have been discovered in IIM subgroups, although further validation in independent cohorts is needed.

References:

[1] Ayoglu B1 et al. Anoctamin 2 identified as an autoimmune target in multiple sclerosis. Proc Natl Acad Sci U S A. 2016 Feb 23;113(8):2188-93.

Disclosure of Interest: None declared

DOI: 10.1136/annrheumdis-2017-eular.5863

\section{FRI0368 NAILFOLD CAPILLAROSCOPY CHANGES REFLECT ENDOTHELIAL ACTIVATION AND INJURY IN PATIENTS WITH SYSTEMIC SCLEROSIS}

A.M. Gheorghiu ${ }^{1}$, R. Sfrent-Cornateanu ${ }^{2}$, D. Marta ${ }^{3}$, M. Bojinca ${ }^{1}$, S. Magda ${ }^{4}$ T. Constantinescu ${ }^{5}$, A. Soare ${ }^{6}$, R. Dobrota ${ }^{6}$, L. Macovei ${ }^{1}$, V. Stoica ${ }^{1}$, C. Bara ${ }^{2}$ C. Mihai ${ }^{1}$. ${ }^{1}$ Internal Medicine and Rheumatology, Cantacuzino Hospital; ${ }^{2}$ Immunology and Physiopathology Department, Carol Davila University of Medicine and Pharmacy; ${ }^{3}$ Victor Babes National Institute of Research and Development; ${ }^{4}$ Cardiology Department, University Emergency Hospital; ${ }^{5}$ Marius Nasta National Pneumology Institute, Carol Davila University of Medicine and Pharmacy; ${ }^{6}$ Internal Medicine and Rheumatology, Cantacuzino Hospital, Carol Davila University of Medicine and Pharmacy, Bucharest, Romania

Background: Systemic sclerosis (SSc) is a severe connective tissue disease characterized by vascular and fibrotic changes in the skin and various internal organs. Pathogenesis of SSc includes early-onset vasculopathy with endothelial cell activation, microvascular injury and impaired angiogenesis.

Objectives: We aimed to determine the association of several biological molecules reflecting endothelial cell activation or dysfunction: E- selectin (E-sel), inter-cellular adhesion molecule 1 (ICAM-1), endothelin 1 (ET-1), von Willebrand factor (vWF) and interleukin 6 (IL-6), with distinct capillaroscopic SSc patterns and with more severe disease.

Methods: Forty consecutive SSc patients attending our EUSTAR SSc clinic, aged [median (IQR)] 52 (18) years, male gender 4/40 (10\%), diffuse cutaneous subset (dcSSc) 14/40 (35\%) were enrolled in this study. Extensive clinical and nailfold capillaroscopy (NFC) pattern assessment, as well as quantification of serum E-sel, ICAM-1, ET-1, VWF, IL-6 and C-reactive protein (CRP) were performed on all patients. Associations between vascular biomarkers and disease characteristics were evaluated by Mann-Whitney U-test and Spearman correlations.

Results: NFC "late" pattern was found in 21 patients, while 6 had "early" and 13 had "active" NFC pattern. All 5 vascular biomarkers correlated with each other good to moderately, with $r$ indices varying between 0.660 and 0.332 , the only exception being ET-1 which did not correlate with E-sel. Good correlations ( $r$ 0.465 to 0.727 ) were also found between all 5 biomarkers and CRP. Patients with severe vasculopathy, as reflected by the NFC "late" pattern, had higher levels of IL-6 (median 12.06 vs. $3.08 \mathrm{pg} / \mathrm{mL}, \mathrm{p}=0.001$ ), ET-1 (median 2.06 vs 1.59 $\mathrm{pg} / \mathrm{mL}, \mathrm{p}=0.029$ ), vWF (median $3284 \mathrm{vs} 2730 \mathrm{IU} / \mathrm{mL}, \mathrm{p}=0.013$ ) and $\mathrm{E}$-sel (median 52.6 vs. $42.3 \mathrm{ng} / \mathrm{mL}, \mathrm{p}>0.05)$, compared to patients with NFC "early" or "active" patterns. There was a significant, negative correlation between lung transfer for carbon monoxide (DLCO) and E-sel, ICAM-1 (both $p<0.001)$ and vWF $(p=0.013$ ). $\mathrm{ET}$-1 was higher in patients with more severe disease (dcSSc, patients positive for anti-topoisomerase antibodies and patients with a history of digital ulcers - all $\mathrm{p}<0.05)$.

Conclusions: Serum biomarkers reflecting endothelial cell activation and/or dysfunction are elevated in patients with more severe SSc-associated vasculopathy and correlate with serum CRP. Together with NFC data they might be used for assessing vasculopathy severity in SSc and identifying patients who would benefit from more aggressive vasoactive treatment.

Acknowledgements: This work was performed as part of the project "Development of a computer-based nailfold videocapillaroscopy (NVC) system for longitudinal evaluation of patients with systemic sclerosis" (QUANTICAP), financed by the UEFISCDI PN-II-PT-PCCA-2013-4-1589 grant.

Disclosure of Interest: None declared

DOI: 10.1136/annrheumdis-2017-eular.5203

\section{FRI0369 PROSPECTIVE EVALUATION OF THE CAPILLAROSCOPIC SKIN ULCER INDEX (CSURI) IN CLINICAL PRACTICE}

U.A. Walker ${ }^{1}$, V.K. Jaeger ${ }^{1}$, L. Arlettaz ${ }^{2}$, M. Banyai ${ }^{3}$, J. Beron ${ }^{4}$, C. Chizzolini ${ }^{5}$, E. Gröchenig ${ }^{6}$, R.B. Mueller ${ }^{7}$, F. Spertini ${ }^{8}$, P. Villiger ${ }^{9}$, O. Distler ${ }^{10}{ }^{1}{ }^{1}$ University Hospital Basel, Basel; ${ }^{2}$ Hôpital du Valais, Sion; ${ }^{3}$ Kantonsspital Luzern, Luzern;

${ }^{4}$ Actelion Pharma Schweiz AG, Baden; ${ }^{5}$ Hôpitaux Universitaires de Genève, Genève; ${ }^{6}$ Kantosspital Aarau, Aarau; ${ }^{7}$ Kantonsspital St. Gallen, St. Gallen; ${ }^{8}$ Centre Hospitalier Universitaire Vaudois, Lausanne; ${ }^{9}$ University Hospital Bern, Bern; ${ }^{10}$ University Hospital Zurich, Zurich, Switzerland

Background: Nailfold videocapillaroscopy (NVC) is an imaging technique representing a reliable tool for the classification, diagnosis and monitoring of systemic sclerosis (SSc) patients. The capillaroscopic skin ulcer index (CSURI) was suggested to identify patients at risk of developing digital ulcers (DU) [1].

Objectives: This study aims (1) to describe the practicality of the CSURI in clinical practice, (2) to describe the change of CSURI during follow-up, and (3) to assess associations between the change in CSURI and demographic and disease characteristics. 
Methods: This Swiss multicentre, prospective, observational study analysed SSc patients with a history of active DU. Demographic characteristics and routine clinical data were recorded and patients underwent NVC at baseline and at the follow-up visit.

The centres' investigators were trained to perform NVC and, the nailfolds of 8 fingers (digit $2-5$ on both hands) were examined if possible. All images were stored centrally and separately analysed by two extensively trained and experienced reviewers (OD \& UAW) separately.

In each NVC image the total number of capillaries in the distal row, the number of megacapillaries and the maximum diameter of the megacapillaries were assessed. Based on these 3 characteristics, the CSURI was calculated according to Sebastiani et al. [1] for patients with at least 1 megacapillary present. Data were analysed descriptively.

Results: Between 2011 and 2015, 61 patients fulfilled the eligibility criteria and had at least one follow-up visit (median observation time 1.0 years, interquartile range [IQR] 1.0-1.1). Of these patients, more than a third (reviewer 1: $n=24$ patients, $39 \%$; reviewer 2 : $n=26$ patients, $43 \%$ ) had no megacapillaries present on NVC on any assessed finger and hence the CSURI could not be calculated for those either at baseline or follow-up. Therefore, this analysis is based on the remaining 34 patients who had at least one megacapillary present on NVC at baseline and at follow-up by both reviewers (26\% male; median age 57 years, IQR 48-65 years).

The median baseline CSURI scores according to reviewer 1 was 5.3 (IQR 2.6-16.3) increasing to a median of $5.9(1.3-12.0)$ at follow-up. The CSURI as evaluated by reviewer 2 reduced from baseline (median 6.4, IQR 2.4-12.5) to follow-up (5.0, IQR 1.7-10.0)

None of the assessed demographic or disease characteristics (Box 1) were associated with the changes in the CSURI between baseline and follow-up for the scores obtained by reviewer 1 and reviewer 2 at the same time; although the limited sample size should be kept in mind interpreting this lack of association.

Box 1: Characteristics assessed for associations with the baseline capillaroscopic skin ulcer index (CSURI) as well as the change in CSURI between the baseline and the follow-up visit.

Demographic characteristics

Age

Sex

Smoking habit (Never/previous/current smoker)

Disease characteristics

Time since Raynaud's phenomenon onset

Time since first non-Raynaud's phenomenon manifestation

Time since first digital ulcers

Previous major digital vascular complication (None/soft tissue infection/

auto-amputation/gangrene)

Cutaneous involvement (Diffuse/limited)

Erectile dysfunction

Proteinuria $(>300 \mathrm{mg} / \mathrm{dl})$

Renal crisis

Pulmonary arterial hypertension

Raynaud's phenomenon condition score at baseline

Modified Rodnan skin score at baseline

Number of digital ulcers at baseline

Laboratory parameters

Anti-nuclear antibodies positive

Anti-centromere antibodies positive

Anti-topoisomerase autoantibodies positive

Conclusions: In this study, around $40 \%$ of patients could not be evaluated with the CSURI due to the absence of megacapillaries on NVC. Clinical decisions based on the CSURI in routine practice should be made with caution, as it can vary greatly between assessors even if they are extensively trained.

\section{References:}

[1] Sebastiani M, et al. Predictive role of capillaroscopic skin ulcer risk index in systemic sclerosis: a multicentre validation study. ARD 2012;71:67-70.

Acknowledgements: This study was supported by Actelion Pharma Schweiz AG.

Disclosure of Interest: U. Walker: None declared, V. Jaeger: None declared, L. Arlettaz: None declared, M. Banyai: None declared, J. Beron Employee of: Actelion Pharma Schweiz AG, C. Chizzolini: None declared, E. Gröchenig: None declared, R. Mueller: None declared, F. Spertini: None declared, P. Villiger: None declared, O. Distler Grant/research support from: 4 D Science, AbbVie, Actelion, Active Biotec, Bayer, Biogenldec, BMS, Boehringer Ingelheim, ChemomAb, EpiPharm, espeRare foundation, Genentech/Roche, GSK, Inventiva, iQone Healthcare, Lilly, medac, Mepha, Medlmmune, Mitsubishi Tanabe Pharma, Pharmacyclics, Pfizer, Sanofi, Serodapharm and Sinoxa, Consultant for: 4 D Science, AbbVie, Actelion, Active Biotec, Bayer, Biogenldec, BMS, Boehringer Ingelheim, ChemomAb, EpiPharm, espeRare foundation, Genentech/Roche, GSK, Inventiva, iQone Healthcare, Lilly, medac, Mepha, Medlmmune, Mitsubishi Tanabe Pharma, Pharmacyclics, Pfizer, Sanofi, Serodapharm and Sinoxa DOI: 10.1136/annrheumdis-2017-eular.1827

\section{FRI0370 THREE-DIMENSIONAL NAILFOLD CAPILLARY IMAGING BY DYNAMIC OPTICAL COHERENCE TOMOGRAPHY IN SYSTEMIC SCLEROSIS: A VALIDATION STUDY USING NAILFOLD VIDEO-CAPILLAROSCOPY}

G. Abignano ${ }^{1,2}$, L. Green ${ }^{1,2}$, S. Eng ${ }^{1,2}$, P. Emery ${ }^{1,2}$, F. Del Galdo ${ }^{1,2} .^{1}$ Leeds Institute of Rheumatic and Musculoskeletal Medicine, University of Leeds; ${ }^{2} \mathrm{NIHR}$ Leeds Musculoskeletal Biomedical Research Unit, Leeds Teaching Hospitals NHS Trust, Leeds, United Kingdom

Background: Optical Coherence Tomography (OCT) of the skin has been proposed as imaging biomarker of fibrosis in Systemic Sclerosis (SSc) (1). However, over the past few years, growing efforts have been made to enable OCT to the study of human skin microcirculation including human nailfold (2). Dynamic OCT (D-OCT) is a newly developed OCT technology that allows detection of blood flow in vivo in addition to the images of traditional OCT scans (3).

Objectives: Aims of this study were: 1) to evaluate face/criterion validity and feasibility of nailfold capillary D-OCT imaging as compared with nailfold videocapillaroscopy (NVC) capillary patterns in SSc patients; 2 ) to investigate whether D-OCT could offer a complementary value to NVC through the 3-dimensional reconstruction and quantification of nailfold capillary abnormalities in SSc patients. Methods: Fifty subjects including forty SSc patients all fulfilling 2013 EULAR/ACR classification criteria (10 with normal/non-specific, 10 with early, 10 with active, 10 with late capillary pattern respectively) and ten age/gender-matched healthy volunteers (HV) were enrolled in this study. All subjects had NVC done on 8 fingers and classified according to the capillary pattern. Nailfold of the finger with the worst capillary score in SSc patients and the 4th finger (the most affected in the SSc groups) of the dominant hand in subjects with normal capillary pattern was subsequently scanned using Vivosight D-OCT (Michelson Diagnostics Ltd., Kent, UK). D-OCT images were analyzed using the proprietary software tool to extract a quantitative measure of the speckle variance (SV)-signal. Results were expressed as mean \pm standard error. Statistical analysis was performed using GraphPad Prism software V.7.0.

Results: The finger with the worst capillary score was the 4th $(83 \%)$ in the SSc patients. The typical nailfold capillary features seen at NVC were visualized at D-OCT images. Representative images are shown in figure 1. Each nailfold D-OCT scan lasted 60 seconds, was well tolerated and did not require use of gel or immersion oil. OCT mean SV-signal measurements were significantly different between HV and SSc patients with any specific capillary pattern $(0.16 \pm 0.02$ vs $0.10 \pm 0.01, p=0.0028)$ and between SSc patients without and with specific capillary pattern $(0.14 \pm 0.01$ vs $0.10 \pm 0.01, p=0.02)$. The mean SV-signal was higher in $\mathrm{HV}$ than in SSc patients without specific capillary pattern however the difference was not statistically significant. When analyzing the three specific capillary patterns, SV-signal measurements were not significantly different. Interestingly, within the late capillary pattern, mean SV-signal was significantly lower in patients displaying capillary loss as main feature compared with those with remarkable neoangiogenesis $(p=0.03)$.

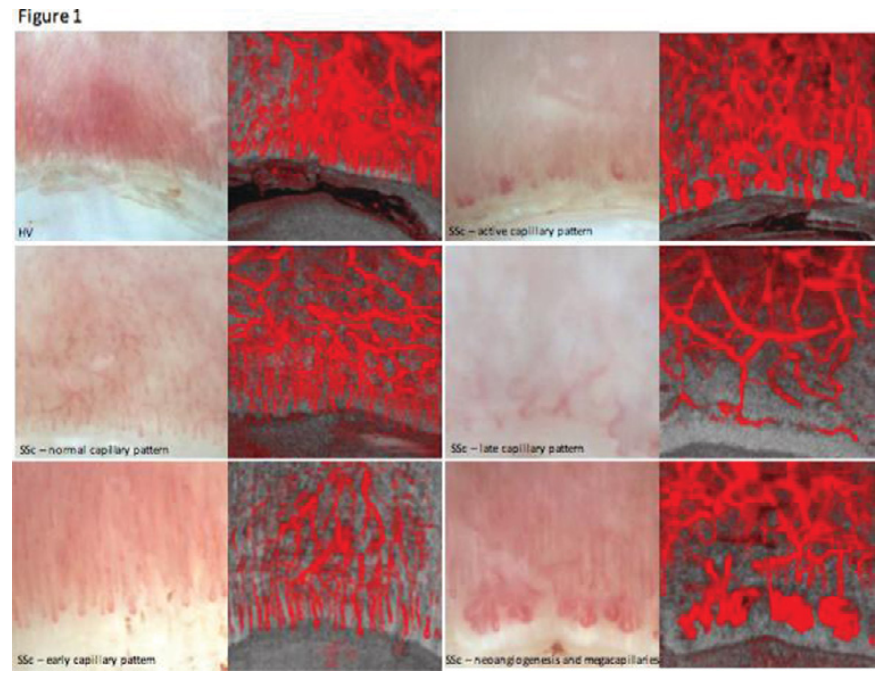

Conclusions: D-OCT is a feasible technique able to reproduce the capillary changes seen at NVC in SSc patients. More importantly D-OCT could offer a complementary value to quantify peripheral blood flow at capillary level. Future longitudinal studies are needed to evaluate the sensitivity to change over time and the potential of D-OCT as quantitative outcome measure of microvasculopathy in SSc.

References:

[1] Abignano G et al. Ann Rheum Dis 2013.

[2] An L et al.Opt Express 2010

[3] Ulrich $\mathrm{M}$ et al.Dermatology 2016.

Disclosure of Interest: None declared

DOI: 10.1136/annrheumdis-2017-eular.5598 